\title{
Nutritional Approach of Pediatric Patients Diagnosed with Congenital Heart Disease
}

\author{
Togănel Rodica \\ II Pediatric Cardiology Clinic, County Emergency Clinical Hospital, Tîrgu Mureș, Romania
}

\begin{abstract}
Congenital heart defects are among the most frequent anomalies present at birth, representing a heterogeneous group of malformations, both in terms of pathogenesis and clinical significance of the lesion. Failure to grow is well documented in infants with complex congenital heart defects; the presence of associated chromosomal abnormalities, cyanosis, and cardiac failure adds to the complexity and challenge. Malnutrition etiology can be grouped into the following three categories: inadequate intake, inefficient absorption and utilization, and/or increased energy needs. The consequences of malnutrition are both short and long term, timely nutritional intervention being necessary in order to maintain an adequate nutritional state. Because there are several types of congenital heart defects and multiple mechanisms by which they produce failure to thrive, no single strategy will be adequate to treat all cases. Medical complications such as chylotorax, necrotizing enterocolitis, laryngeal and neurological dysfunction play a major role in the requisite nutrition therapy in infants with congenital heart defect; limited access to human milk and parenteral concerns, as well as stress about feeding are also factors that can contribute to poor outcomes concerning nutrition and growth. Protocols are being considered and designed, and a systematic approach is always needed. The quality of life for patient and family, as well as getting the child back on track for age-appropriate development are always at the fore-front of each care plan.
\end{abstract}

Keywords: congenital heart disease, nutrition

Received: 20 November 2012

\section{Introduction}

Congenital heart disease (CHD) represents a heterogeneous group of malformations, both in terms of pathogenesis and clinical significance of the lesion, defined by Mitchell et al. as structural abnormalities of the heart and great vessels, present at birth, that have, or will have a functional response at some point in life [1]. CHDs are among the most frequent anomalies present at birth, with a reported incidence of 8/1000 live births, with high impact on neonatal morbidity and mortality (33\%) [2].

Infants with CHD are usually born at full term, with a weight that is appropriate for the gestational age. Nutritional issues often emerge shortly after birth, and growth failure becomes apparent in the neonatal period, if hemodynamically significant CHD is present. In some instances, the delay can be relatively mild, whereas in other cases, the failure to thrive can result in permanent physical or developmental impairment. The degree and type of malnutrition may be related to the $\mathrm{CHD}$ characteristics, including the presence of cyanosis, congestive heart failure or pulmonary hypertension. Infants with cyanosis tend to have similar alterations in both weight and length, while infants with acyanotic lesion tend to have more pronounced decreases in weight gain velocity as compared to length $[3,4]$. Descriptions of growth status in acyanotic and cyanotic lesions have changed over the past 25 years, as surgical intervention now occurs earlier in infancy for children with cyanotic lesions. As such, recent studies have shown an

Correspondence to: Rodica Togăne

E-mail: rodicatoganel@yahoo.com emergence of more pronounced growth retardation and wasting in acyanotic infants [5].

Feeding, the most complex task the infant must perform, requires coordination and integration of motor and sensory pathways of the oropharynx. Abnormalities in swallowing in these infants include variability in sucking, an uncoordinated suck, swallow and breathing, and oral transit time. Poor skills in feeding and any dysfunctional swallowing can directly affect the ability to feed, and hence the infant's achievement of an adequate nutritional state and growth [6].

In consequence, failure of growth in itself is associated with an array of health problems, its cause being multifactorial and directly related to challenges encountered with intake of substrate for energy.

\section{Etiology of malnutrition}

Children with CHD face many challenges to achieve optimal short-term and long-term growth, and the presence of associated chromosomal abnormalities, cyanosis, and cardiac failure adds to the complexity and challenge.

The etiology of malnutrition in the pediatric patient diagnosed with $\mathrm{CHD}$ can be grouped into the following three categories: inadequate intake, inefficient absorption and utilization, and/or increased energy needs. Any or all of these factors may be present in a child with CHD, making the delineation of optimal nutrition and medical therapy difficult. In addition, the association of genetic defects, prematurity or any other congenital or acquired medical issues further complicates the nutritional management of these patients. 


\section{Inadequate energy intake}

Decreased caloric intake has been shown to be the most important cause of malnutrition in pediatric patients with CHD [7]. A study published by Hansen and Dorup [8] showed that caloric intake in patients with $\mathrm{CHD}$ was $76 \%$ that of normal age-matched controls. Fatigue upon feeding may represent a possible cause to explain the decreased intake $[7,9]$. Chronic hypoxia leads to both dyspnea and tachypnea during feeding, causing the child to tire easily and thus reduce the quantity of food consumed.

\section{Inefficient absorption and utilization}

Intestinal dysfunction caused by reduced blood flow to and from the splanchnic circulation is often encountered in patients with $\mathrm{CHD}$, leading to malabsorption. This suggests that even children with $\mathrm{CHD}$ whose caloric intake is normal for their age may not be receiving enough calories to achieve normal weight. It has to be mentioned that there is still considerable controversy regarding the role of malabsorption in growth disturbances, therefore this mecanism should be considered until further research is presented $[7,9,10]$.

\section{Increased energy expenditure}

A study published in 2002 [11] showed that infants with failure to thrive due to ventricular septal defects have a $40 \%$ elevation in total energy expenditure (TEE). Surprisingly, resting energy expenditure (REE) was found to be the same between control and children with ventricular septal defects. The difference between REE and TEE was 2.5 times higher in the ventricular septal defect group than the control, indicating that their energy during activity is much higher.

The body composition of children with CHD modifies, by having an elevated percentage of lean body mass, compared with fat deposition. This is explained by the decreased caloric intake and greater energy expenditure encountered in these children, having in consequence less energy available for fat deposition. The increased percentage of lean body mass tends to increase the basal metabolic rate [12], further increasing metabolic rate, which, if left untreated, can dramatically worsen the child's overall health status.

\section{Consequences of malnutrition}

Malnutrition has both short and long term consequences. In the short term, infants and children with pre-operative malnutrition have a reduced ability to fight infection or to heal optimally from surgical wounds $[13,14,15]$, thereby prolonging intensive care unit and total hospital stay for post-operative CHD infants. In the long term, malnutrition in infancy can produce suboptimal growth and physical and cognitive development later in childhood and adolescence. It was shown by clinical studies that infants operated on earlier, experience more significant catch-up growth in weight and length, having a better chance for achieving maximal genetic potential [16]. Delayed puberty is possible in children with impaired linear growth, as puberty does not typically begin until the child reaches the height of a normal 12-13-year old [13,17]. Another study showed that children, especially under 3 years of age and with a cyanotic lesion, tend to have lower than normal intelligence scores, which could be a result of alterations in brain weight, yielding reduced cognitive functioning [17].

Studies in developed countries have shown normalization of somatic development after early surgical correction of CHD. In developing countries, due to limited material resources, corrective surgery is performed later, which leads to a vicious cycle of heart failure and respiratory infections. This predisposes to an increased prevalence of preoperative malnutrition in children with CHD [18].

\section{Management of growth disturbances}

While timely nutritional intervention is necessary to maintain an adequate nutritional state, finding strategies that enhance feeding skills and ensure an adequate nutritional intake has eluded both professionals working in pediatric cardiology and colleagues working in primary care. Many pediatric cardiac centers are striving to develop a protocol for feeding after cardiac surgical procedures that is efficient and effective in ensuring an adequate intake of substrates for energy, that fosters a gain in weight and allows for an objective assessment of the skills with feeding and progress achieved by the infant. Protocols are being considered and designed and a systematic approach is always needed [6].

Based on the premise that inadequate caloric intake is the leading cause of growth failure in children with $\mathrm{CHD}$, it seems logical that increasing the number of consumed calories would have a positive effect on growth and development. Unger et al. showed that dietary intervention including nutritional analysis and counseling increased mean intake from $90 \%$ to $104 \%$ of the RDA for calories and increased weight from $83.1 \%$ to $88.3 \%$ of ideal body weight [19]. In consequence, because counseling alone increased the caloric intake and weight in these children, parental education from a dietitian plays an important role in optimizing their feeding.

Even with nutritional counseling, some children with $\mathrm{CHD}$ are incapable of consuming larger quantities of food due to the high energy consumption that eating presumes. Authors like Jackson and Poskitt have proposed supplementing formula or breast milk with glucose polymers, increasing mean energy intake by $31.7 \%$ and resulting in a weight gain improvement from $1.3 \mathrm{~g} / \mathrm{kg} /$ day in controls to $5.8 \mathrm{~g} / \mathrm{kg} /$ day with high energy feeding [20]. This method has its drawbacks, because another study found that feeding malnourished children high-energy formula may stimulate greater diet-induced thermogenesis and increase metabolic inefficiency, canceling some of the positive effect [12]; for these children it can also be difficult to tolerate the concentrated glucose, thereby they need close monitoring.

Unfortunately, there are cases when despite the most aggressive feeding programs, children are still unable to 
ingest enough calories in order to achieve or maintain a normal body weight. In these cases it may become advisable to provide direct gastric nutritional support using percutaneous endoscopic gastrotomy (PEG). The advantage of this method relies in the fact that the child does not need to expend any energy to feed, therefore more is available for growth. One study has shown that 12 newborn patients of the 13 studied, diagnosed with CHD, who were fed using PEG, had normal growth rates for their age, suggesting that PEG could be used to prevent growth disturbances in newborns with CHD [21]. In consequence, prophylactic enteral nutrition may be indicated in patients with CHD even before growth disturbances become apparent $[21,22]$.

\section{Breast feeding or bottle feeding?}

The decision of whether to breast or bottle feed an infant is very important for both the child and the parents. The benefits of breast feeding are well known for both the child and the mother, the most important of all being that it helps foster the bond between mother and child, while providing the best source of nutrients. Surprisingly, many parents opt to bottle feed their children, considering that breast feeding may be too difficult for children with CHD. They believe that by using the bottle, they can control the flow rate and therefore deliver more volume with less effort. In order to study the advantages and potential disadvantages of breast feeding compared to bottle feeding, Marino et al. discovered that oxygen saturation in infants with CHD was lower in the group that was bottle feeding than in the group that was breast feeding $(\mathrm{p}<0.0001)$, indicating that it is less stressful for the infant to breast feed than bottle feed [23]. In this case, it is important to consider a potential contribution of non-cardiac factors, such as parent-child interaction during feeding, by giving the mothers of children with CHD additional support and education in breast feeding techniques to reduce stress and increase success.

The answer to this question remains a challenge, because breast feeding exclusively in children with CHD contradicts other studies that have shown increased weight gain in children on high energy supplemented breast milk or formula [20].

Based on the study published by Marino et al. [23], choosing between breast and bottle feeding depends heavily on the type of cardiac lesion. For example, in infants with cyanotic lesions, because keeping their oxygen saturation as high as possible represents the most important goal, it may be necessary to breast feed at the expense of energy needs. Conversely, in children with acyanotic lesions it may be perfectly acceptable to bottle feed, as this will have little adverse effect on oxygen saturation.

\section{Surgical correction}

Most CHDs require surgical correction at some point, and the timing of the surgery depends not only on the type of the cardiac lesion, but also on the child's weight. Surgical correction is performed in most cases as soon as possible, after the child reaches ideal weight. Multiple studies published in the international literature showed improvement of the $\mathrm{Z}$ scores for weight and height after surgery [18]. The explanation given by two recent studies was that surgical correction of the cardiac defect lowers energy expenditure in these children, one of the main causes of growth failure [24]. In consequence, surgical correction represents a therapeutic measure for failure to thrive in children with $\mathrm{CHD}$, and must be indicated when all nutritional measures have been exhausted, despite increased morbidity and mortality associated with cardiac surgery in underweight infants [10].

Nutrition remains an important factor to be followed after the surgery, as well. In complex CHD, after surgery, there might be a period when only parenteral and/or enteral nutrition is indicated. Parenteral nutrition is indicated when the expected time to begin and achieve adequate enteral nutrition is more than $3-5$ days $[13,25]$ or when the gastrointestinal tract cannot be used for other reasons besides cardiac complications, such as other congenital anomalies involving the gut. Enteral nutrition should be commenced as soon as it is medically feasible, in order to reduce the risk of gut atrophy and loss of the intestinal mucosal barrier, which increases the risk of bacterial translocation and bacteremia $[13,26,27]$. Enteral nutrition can be delivered using a nasogastric tube or a small bowel tube, noting that in case of a longer estimated period than 6-8 weeks for a feeding tube to be needed for nutrition, a gastrostomy tube should be considered.

\section{Nutrition management of complications and comor- bidities}

CHD and cardiac surgery are often associated with nutrition-related complications and comorbidities: laryngeal dysfunction, acute renal failure, necrotizing enterocolitis, chylothorax and protein-losing enteropathy being the most encountered ones [26,28-35].

Laryngeal dysfunction has an incidence of $1.7-9 \%$ after cardiac surgery, and it may be due to operative trauma to the recurrent laryngeal nerve, prolonged intubation or neurological injury. Management of these cases includes thickening feeds to nectar or honey consistency, feeding positioning (right side down), and/or supplemental/sole tube feeding [26,28-35].

Acute renal failure occurs in $1-42 \%$ after cardiac surgery, and it is caused by ischemic and inflammatory insults occurring during cardio-pulmonary bypass (the longer the bypass times, the higher the risks). Nutrition measures to be considered in these cases are: fluid restriction, concentrated formula caloric density or parenteral nutrition, using formulas low in electrolytes, and in case of dialysis - supplement with water soluble vitamins and trace elements, providing 2-3 $\mathrm{g} / \mathrm{kg} /$ day protein for continuous renal replacement therapy and $4 \mathrm{~g} / \mathrm{kg} /$ day for peritoneal dialysis [26,28-35]. 
Necrotizing enterocolitis has an incidence of 3.3-6.8\% after cardiac surgery, and it may be secondary to poor gut perfusion due to diastolic flow reversal in the superior mesenteric artery, particularly in single ventricle physiology, deep hypothermia during cardio-pulmonary bypass causing decreased gut perfusion or proinflammatory response to $\mathrm{CHD}$ and cardio-pulmonary bypass [14]. Measures in these cases are as follows: provision of adequate parenteral nutrition if feeds are held, and when feeds are restarted, use of hypoallergenic infant formula [26,28-35].

Chylothorax occurs in $2.5-4.7 \%$ after cardiac surgery and is most often due to the injury of the thoracic duct during surgery, thrombosis of the superior vena cava resulting in occlusion, or to the elevated central venous pressure following surgery. Nutrition measures to be followed in these cases are: limit dietary long-chain triglycerides for up to 6 weeks after surgery by offering very high medium-chain triglycerides formula for infants or a diet very low $(<10 \mathrm{~g} /$ day $)$ in total fat for toddlers and children; breast milk can be skimmed with a centrifuge, then fortified with high medium-chain triglycerides formula or oil; ensure $2-4 \%$ of calories from long chain fat to prevent essential fatty acid deficiency; ensure adequate protein, electrolyte, and vitamin intake, as nutrients are lost in chyle drained via chest tube; parenteral nutrition may be required in cases refractory to nutrition and medical management [26,28-35].

Protein-losing enteropathy (PLE) is a disorder that appears as a complication of Fontan procedures, and is characterized by abnormal and often profound enteric protein loss. Fontan circulation is a procedure used in palliation of complex $\mathrm{CHD}$, and represents a total cavopulmonary anastomosis, meaning that the systemic venous blood flows to the lungs without passing through a ventricle. PLE is a relatively rare complication. In an international multicentre study, involving 35 centres and 3029 patients with Fontan repair between 1975 and 1995, PLE occurred in 114 patients - 3.8\% [36]. While some progress in understanding the PLE epidemiology and prognosis has been made in the last decades, accurate data about its pathogenesis and pathophysiology remain limited. Possible causes of PLE are as follows: problems in the Fontan circuit (gradient at connection, poor design), problems of the pulmonary vasculature (increased pulmonary vascular resistance, hypoplasia, distorsion), cardiac problems (atrioventricular valve regurgitation, myocardial dysfunction). Therapeutic measures in PLE are as follows: non-specific medical therapy, consisting in diets high in protein and low in fat, anti-congestive cardiovascular medical regimens, periodic albumin infusions and specific medical therapy, including the use of unfractionated heparin, corticosteroids, transcatheter therapy (dilation and/or stenting of obstructions in blood flow, coil embolisation of significant aorto-pulmonary collaterals, fenestration of the circuit), heart transplant. Despite improvement in therapies used in Fontan patients with
PLE, their prognosis has not significantly improved and mortality related to PLE remains high [37].

\section{Conclusion}

Failure to thrive represents a serious, well documented problem in children with $\mathrm{CHD}$, proving to be a challenge for the clinician. Along with medical and surgical treatment of these patients, developing a nutritional strategy plays a key role in the management of cardiac malformations. Because there are several types of $\mathrm{CHD}$ and multiple mechanisms by which they produce failure to thrive, no single strategy will be adequate to treat all cases. There is great need for a systematized approach to feeding that incorporates physiological, neurological, and behavioral assessments, including a concerted effort between parents, physicians, nurses and other healthcare professionals to develop a plan that will be appropriate on an individual basis. The quality of life for patient and family, as well as getting the child back on track for age-appropriate development, is always at the fore-front of each care plan. Further studies are needed to identify the most effective therapeutic measures in managing various causes of growth failure.

\section{References}

1. Mitchell SC, Korones SB. Berendes HW. Congenital heart disease in 56,109 births. Incidence and natural history, Circulation. 1971;43:323332.

2. Schultz AH, Localio AR, Clark BJ, et al. Epidemiologic features of the presentation of critical congenital heart disease: Implantations for Screening. Pediatrics. 2008;121:751-757.

3. Norris MK, Hill CS. Nutritional issues in infants and children with congenital heart disease. Crit Care Nurs Clin North Am.1994;6:153-163.

4. Leitch CA. Growth, nutrition and energy expenditure in pediatric heart failure. Prog Pediatr Cardiol. 2000;11:195-202.

5. Peterson RW, Wetzel GT. Growth failure in congenital heart disease: where are we now? Curr Opin Cardiol. 2004;19:81-83.

6. Medoff-Cooper B, Irving Sh. Innovative strategies for feeding and nutrition in infants with congenitally malformed hearts. Cardiology in the Young. 2009;19(suppl. 2):90-95.

7. Varan B, Tokel K, Yilmaz G. Malnutrition and growth failure in cyanotic and acyanotic congenital heart disease with and without pulmonary hypertension. Arch Dis Child. 1999;81(1):49-52.

8. Hansen SR, Dorup I. Energy and nutrient intakes in congenital heart disease. Acta Paediatr. 1993;82(2):166-72.

9. Leitch CA. Growth, nutrition and energy expenditure in pediatric heart failure. Prog Pediatr Cardiol. 2000;11(3):195-202.

10. Poskitt EM. Failure to thrive in congenital heart disease. Arch Dis Child. 1993;68(2):158-60.

11. Ackerman IL, Karn CA, Denne SC, Ensing GJ, Leitch CA. Total but not resting energy expenditure is increased in infants with ventricular septal defects. Pediatrics. 1998;102(5):1172-7.

12. Menon G, Poskitt EM. Why does congenital heart disease cause failure to thrive? Arch Dis Child. 1985;60(12):1134-9.

13. Leitch CA. Growth, nutrition and energy expenditure in pediatric heart failure. Prog Pediatr Cardiol. 2000;11:195-202.

14. Poskitt EM. Food, growth and congenital heart disease. Nutr Health. 1987;5:153-161.

15. Anderson JB. Kalkwarf HJ, Kehl JE, et al. Low weight-for-age z score and infection risk after the Fontan procedure. Ann Thorac Surg. 2011;91:14601466.

16. Parrish CR. Nourishing little hearts: nutritiional implications for congenital heart defects. Practical Gastroenterology. 2011;11-34

17. Ehlers $\mathrm{KH}$. Growth failure in association with congenital heart disease. Pediatr Ann. 1978;7:750-759.

18. Vaidyanathan B, Kumar RK. What Determines Nutritional Recovery in Malnourished Children After Correction of Congenital Heart Defects? Pediatrics. 2009;124(2):e294. 
19. Unger R, DeKleermaeker M, Gidding SS, Christoffel KK. Calories count. Improved weight gain with dietary intervention in congenital heart disease. Am J Dis Child. 1992;146(9):1078-84.

20. Jackson M, Poskitt EM. The effects of high-energy feeding on energy balance and growth in infants with congenital heart disease and failure to thrive. Br J Nutr. 1991;65(2):131-43.

21. Bougle D, Iselin M, Kahyat A, Duhamel JF. Nutritional treatment of congenital heart disease. Arch Dis Child. 1986;61(8):799-801.

22. Hofner G, Behrens R, Koch A, Singer H, Hofbeck M. Enteral nutritional support by percutaneous endoscopic gastrostomy in children with congenital heart disease. Pediatr Cardiol. 2000;21(4):341-6.

23. Marino BL, O'Brien $P$, LoRe $H$. Oxygen saturations during breast and bottle feedings in infants with congenital heart disease. J Pediatr Nurs. 1995;10(6):360-4.

24. Mitchell IM, Davies PS, Day JM, Pollock JC, Jamieson MP. Energy expenditure in children with congenital heart disease, before and after cardiac surgery. J Thorac Cardiovasc Surg. 1994;107(2):374-80.

25. Forchielli ML, McColl R, Walker WA, et al. Children with congenital heart disease: a nutrition challenge. Nutr Rev.1994;52:348-353.

26. Owens JL, Musa N. Nutrition support after neonatal cardiac surgery. Nutr Clin Pract. 2009;24:242-249.

27. Hagau N, Culcitchi C. Nutritional support in children with congenital heart disease. Nutr Ther Metab. 2010;28:172-184.

28. Medoff-Cooper B, Naim M, Torowicz D, et al. Feeding, growth, and nutrition in children with congenitally malformed hearts. Cardiol Young. 2010;20 Suppl3:149-153.
29. Giannone PJ, Luce WA, Nankervis CA, et al. Necrotizing enterocolitis in neonates with congenital heart disease. Life Sci. 2008;82:341-347.

30. Cabrera AG, Prodhanb P, Bhuttab AT. Nutritional challenges and outcomes after surgery for congenital heart disease. Curr Opin Cardiol. 2010;25:88-94.

31. Sachdeva R, Hussain E, Moss MM, et al. Vocal cord dysfunction and feeding difficulties after pediatric cardiovascular surgery. J Pediatr. 2007; 151:312-315.

32. Skinner ML, Halstead LA, Rubinstein CS, et al. Laryngopharyngeal dysfunction after the Norwood procedure. J Thorac Cardiovasc Surg. 2005;130:1293-1301

33. Lessen R. Use of skim breast milk for an infant with chylothorax. Infant, Child, and Adolescent Nutrition. 2009;1:303-310.

34. McCray S, Parrish CR. When chyle leaks: nutrition management options. Practical Gastroenterology. 2004;28:60.

35. Li S, Krawczeski CD, Zappitelli M, et al. Incidence, risk factors, and outcomes of acute kidney injury after pediatric cardiac surgery: a prospective multicenter study. Crit Care Med. 2011;39:1493-1499.

36. Mertens L, Hagler D, Sommerville J, et al. Protein losing enteropathy after the Fontan operation: an international multicenter evaluation. J Thorac and Cardiovasc Surg. 1998;115:1063-73.

37. Meadows J, Jenkins K. Protein-losing enteropathy: integrating a new disease paradigm into recommendations for prevention and treatment. Cardiol in the Young. 2011;21:363-367. 